\title{
Kindererziehungszeit
}

\section{Gericht schafft Klarheit für Vertragsärzte}

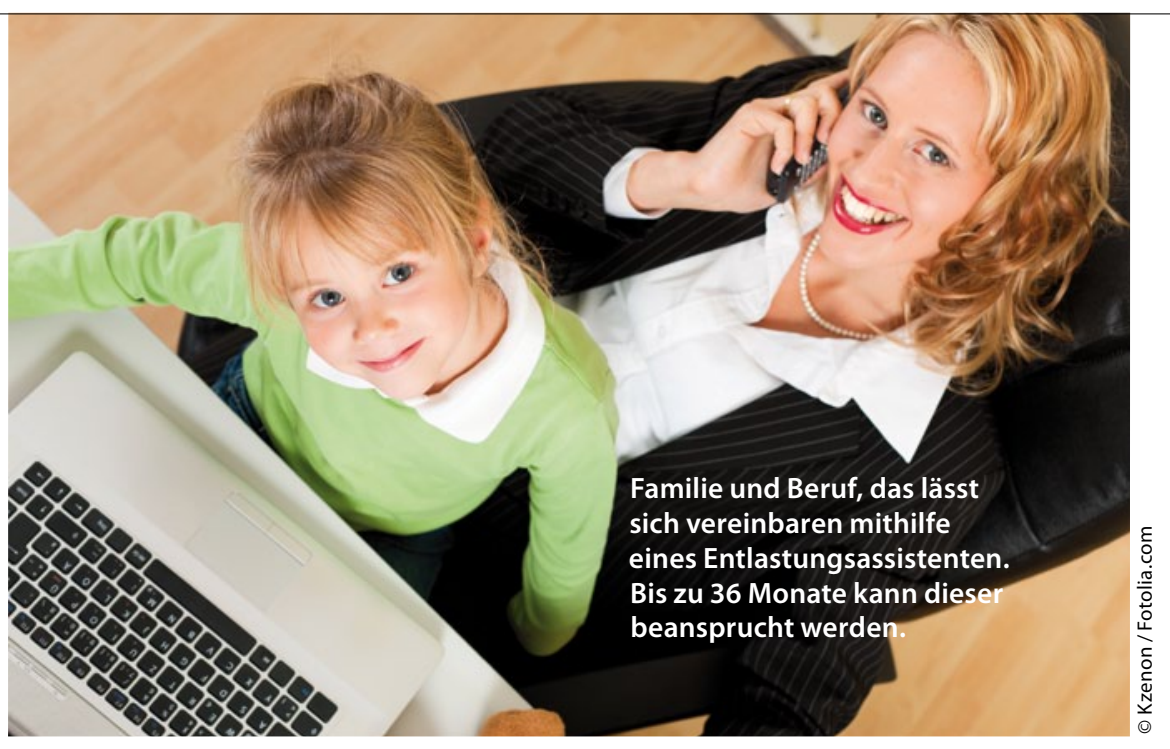

\author{
Vertragsärzte haben einen gewissen Spielraum bei der Beschäftigung \\ von Entlastungsassistenten in der Zeit der Kindererziehung. Dafür \\ hat jetzt ein Landessozialgericht gesorgt - und eine restriktive KV \\ in die Schranken verwiesen.
}

V ertragsärzte können bis zu 36 Monate einen Entlastungsassistenten beschäftigen, wenn sie sich währenddessen der Kindererziehung widmen wollen. Diese Regelung ist 2012 mit dem GKV-Versorgungsstrukturgesetz in Kraft getreten ( $\$ 32$ Abs. 2 der Zulassungsverordnung für Ärzte, Ärzte-ZV). Die Beschäftigung eines solchen Entlastungsassistenten bedarf aber der Genehmigung der zuständigen Kassenärztlichen Vereinigung (KV).

\section{Neue Beschlusslage gemäß Landessozialgericht NRW}

Eine KV vertrat Ende 2012 die Auffassung, die zeitliche Beschränkung von 36 Monaten beziehe sich auf das Lebensalter des Kindes. Ein Entlastungsassistent könne demnach also nur bis zur Vollendung des dritten Lebensjahres genehmigt werden, so die KV. Dem ist das Landessozialgericht Nordrhein-Westfalen (LSG) in einem Eilverfahren mit Beschluss vom 27. Februar 2013 (L 11 KA 8/13 B ER) nicht gefolgt. Der Zeitraum von 36 Monaten bezieht sich laut Gericht auf die Dauer der Vertretung, nicht auf das Lebensalter des Kindes. Der zulässige Zeitraum der Assistentenbeschäftigung ist zwar auf 36 Monate beschränkt. Dieser Zeitraum muss aber nicht zusammenhängend in Anspruch genommen werden. Aus diesem im Gesetz enthaltenen Halbsatz leitet das LSG ab, dass es nicht auf das Lebensalter des Kin- des ankommen kann. Das Gericht wies die Auffassung der KV mit dem Satz zurück, ein „nicht zusammenhängend genommenes Lebensalter sei schwerlich vorstellbar", was für den Humor der Sozialrichter spricht. Damit stärken die Richter die Vereinbarkeit von Beruf und Familie für Vertragsärzte. Die Möglichkeit der Vertretung im Zusammenhang mit einer Entbindung nach $₫ 32$ Abs. 1 Ärzte-ZV haben nur Ärztinnen, die Zulässigkeit der Beschäftigung eines Entlastungsassistenten für die Erziehung ist im Gesetz dagegen geschlechtsneutral formuliert. Begrenzt wird der Zeitraum, in dem Vertragsärzte einen Entlastungsassistenten beschäftigen können nur durch die Merkmale „Kind“ und „Zeit der Erziehung". Der Assistent muss demnach den Praxisinhaber in "Zeiten der Erziehung" entlasten. Die Darstellung, dass man sich der Erziehung des Kindes widmen möchte, dürfte jedoch einfach sein, wenn man z. B. einen Assistenten über 20 Wochenstunden beantragt, um sich etwa am Nachmittag dem Kind zu widmen.

Es stellt sich dann die Frage, ob man einen Assistenten z. B. auch an jedem Freitag beschäftigen kann, um sich freitags der Erziehung des Kindes zu widmen. Dies dürfte zwar etwas begründungsbedürftiger sein, es ist aber auch in diesem Fall nachvollziehbar, dass man sich an einem Wochentag intensiv um sein Kind und die Erziehung kümmern möchte.
Ob der 36-Monats-Zeitraum tageweise zu zählen ist, erscheint dagegen zweifelhaft. Da 36 Monate nicht zusammenhängend genommen werden müssen, wären dies, vereinfacht berechnet, 1.080 Tage. Dies entspräche einem Tag pro Woche für die Dauer von ungefähr 20 Jahren. Eine solche Auslegung dürfte aber mit dem Wortlaut und der Intention des Gesetzes nicht vereinbar sein. Anderenfalls hätte der Gesetzgeber die Beschäftigung eines Assistenten für 144 Wochen oder 1.080 Tage erlauben können, was er aber nicht getan hat. Vertragsärzte werden daher davon ausgehen müssen, dass zwar die 36 Monate nicht zusammenhängend genommen werden müssen, dass aber eine „Stückelung" in kleinere Zeitintervalle als einen Monat nicht zulässig ist. Wenn Praxisinhaber also einen Assistenten tageoder wochenweise beschäftigen, so zählt dies als Beschäftigung während eines Monats.

Bis zu welchem Alter eine Person noch als Kind im Rechtssinne gilt wird in den deutschen Gesetzen nicht einheitlich definiert. Es wird entweder von der Vollendung des 14. Lebensjahres oder des 18. Lebensjahres ausgegangen. Im Alter zwischen 14 und 18 bleibt also Raum für denkbare Auseinandersetzungen über die Beschäftigung eines Entlastungsassistenten, bis zum 14. Geburtstag des Kindes kann jeder Vertragsarzt und jede Vertragsärztin einen solchen Assistenten anstellen, wenn man sich während der "gewonnenen" Zeit der Erziehung des Kindes widmen möchte.

Dr. Ingo Pflugmacher,

Fachanwalt für Medizin- und Verwaltungsrecht, Partner der Kanzlei Busse \& Miessen, Bonn 